\title{
A new Look into the Imperialist Competitive Algorithm
}

\author{
Meisam Booshehri ${ }^{1}$, Peter Luksch ${ }^{2}$ \\ \{meisam.booshehri, peter.luksch\}@uni-rostock.de \\ Institute of Computer Science, University of Rostock, Germany ${ }^{1,2}$
}

\begin{abstract}
Genetic Algorithm is currently used as a solution to various problems in a wide range of disciplines. In order to improve the convergence rates of Genetic Algorithms, a new branch of evolutionary computation called "cultural algorithms" has been introduced that provides the possibility of exchanging in-formation in the population component of a conventional genetic algorithm. As expected in some applications the convergence rates obtained by cultural algo-rithms such as Imperialist Competitive Algorithm (ICA) were better or at least similar to those obtained by applying the genetic algorithms. In this paper, we aim to propose a new perspective, which is assumed to increase the capability of exchanging information in the population component of the evolutionary al-gorithms by providing an infrastructure for dialogues. In other words, we divide a population into several regions equivalent to the empires in ICA where instead of the competition among regions we introduce the notion of Dialogue among regions, which is assumed to improve the convergence rate towards the absolute minimum.
\end{abstract}

Keywords: Imperialist Competitive Algorithm, Evolutionary Computation, Dialogue infrastructures, convergence rate

\section{$1 \quad$ Background and Related work}

Genetic evolution is a process that has been realized after millions of years; however, by simulating and modeling this process, powerful algorithms have been proposed, among them Genetic Algorithm (GA), which is widely used in different disciplines [1]. Another type of evolution has been occurred in human beings' world - cultural and social evolution. Obviously, cultural, social and intellectual evolution of mankind would happen much faster than physical and genetic evolution. Accordingly, such a fast evolution has been considered to develop new branches of evolutionary computation. Cultural algorithms [2] are the beginning of this category that can be seen as an extension for a conventional genetic algorithm since they add the capability of cultural evolution to the previous algorithms in order to improve the rate of con-vergence towards the absolute minimum. In other words, they provide the possibility of exchanging information among the members of the population resulting in proba- 
bly faster convergence rates. For instance, Imperialist Competitive Algorithm (ICA) [3] has attracted a lot of attention in the last years. Comparing against GA, ICA has obtained similar or better results in various applications [4-7]. ICA is started with a population of countries which are equivalent to the chromosomes in GA. Countries are divided into two categories: imperialists and colonies. Imperialist competition for the occupation of colonies and assimilation of them into the imperial society are the core policies of the ICA, which makes the countries move towards the absolute mini-mum. The world's end from the perspective of this algorithm is a unipolar world, which contains just one Imperialist while each colony has a power near to the imperi-alist's power [3]. This works; however, we assume what the ICA portrays as the world's end might differ from the reality since while an imperialist exists in the world it means that somewhere in the world democracy has been violated. Obviously, lack of democracy would lead to conflicts and disputes that would change the world again. From this perspective, what ICA portrays cannot be logically the world's end. We believe in the idea of Dialogue among Civilizations ${ }^{1}$, and we predict that after so many disputes, the mankind would come to this idea finally. Some strategic objec-tives of this idea include: (1) promoting and facilitating the peaceful resolution of conflicts and disputes and (2) reconciling tensions between cultures, countries and religions. By this approach there would be the Utopia where there are only justice and human dignity. There would be a world that moves much faster than the world of imperialists and colonies in almost any scope including cultural, social, intellectual and technological ones. This is another type of evolution that can happen in human beings' world. Accordingly, we are motivated to answer the following question: "Can we make use of the notion of dialogue among civilizations and its consistent evolution to improve the rate of convergence towards the absolute minimum in comparison to the rates obtained by current evolutionary algorithms?"

\section{Proposed idea}

In order to propose our main idea, we start with a fundamental question: "How about designing an evolutionary algorithm whose preliminary idea is to increase the interaction between the members of the population?" To this end, we are wondering of dividing the population into some logical regions -equivalent to the empires in ICA- in which instead of imperialist competitions we make use of constructive dia-logues among the regions to improve the rate of convergence towards the absolute minimum both in serial and parallel kind of the algorithm. After iterative dialogues between region pairs, the mean of members' fitness in each pair should move towards the absolute minimum. To pursue dialogues, a suitable infrastructure is needed to connect the regions with each other. We assume two sample infrastructures to realize our perspective: Linear Infrastructure and Majority-based infrastructure. Besides, we discuss some operators in order to form the core policy of our proposed method.

1 http://en.wikipedia.org/wiki/Dialogue_Among_Civilizations 


\subsection{Dialogue Infrastructures}

We introduce two types of infrastructures for dialogues including linear infra-structure and majority-based infrastructure. Linear infrastructure is our proposal and majority-based infrastructure is a notion which has been used previously in the mutual exclusion algorithms for distributed systems.

- Linear infrastructure: Let $R_{i}$ be the set of members in ith region in the population. A linear infrastructure would be $\bigcup_{i=1}^{n} R_{i}$ where $\forall i: 1 \leq i \leq n:: R_{i} \cap$ $R_{i+1} \neq \varnothing$.

- Majority-Based infrastructure: Simple majorities are quorums[8] and any two sets whose sizes are simple majorities must have at least one element in common. In other words, quorums have the property that any two groups have a non-empty intersection. For instance, as depicted in Fig. 1 among 12 nodes the majority is 7. To discuss it more mathematically, let $R_{i}$ be the set of members in ith region in the population component. A Quorum-Based infrastructure would be $\bigcup_{i=1} R_{i}$ where $\forall i \forall j: 1 \leq i, j \leq n:: \quad R_{i} \cap R_{j} \neq \varnothing$. A common type of a quorum-model is a grid quorum in which the nodes are arranged in a logical grid. Then a quorum is defined as all of a row and all of a column. Quorum size would be $[\sqrt[2]{N-1}\rfloor$ where $\mathrm{N}$ is the number of all the nodes. A sample of grid quorum has been depicted in Fig. 2.

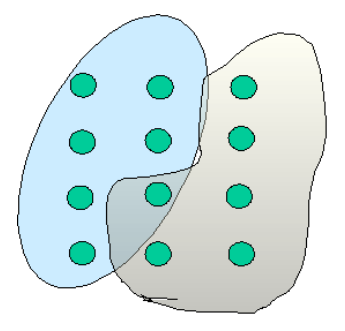

Fig. 2. A sample of determining majority

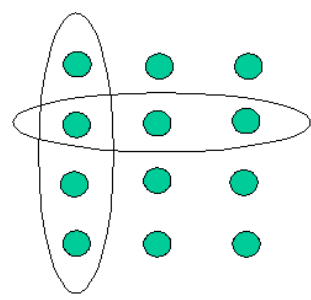

Fig. 1. A sample of Grid Quorum with $\mathrm{N}=12$

\subsection{Proposed operators and policies}

With the new perspective and the sample dialogue infrastructures, the following operators seem to be suitable to form the core policy of a new algorithm.

- Fitness Improver. This operator can be selected among previous operators in GA and ICA, including the mutation operator, cross-over operator and $\alpha$-rotation operator. Also we can make use of a hybrid operator.

- Constructive Dialogue: This operator can be seen as a policy as well. This operator could propagate the high quality of a region into its neighbor regions. By this 
approach, when a region becomes stronger in its mean value, its neighbors' mean value would be increased as well in a way that a much faster convergence rate is provided towards the absolute minimum. Suppose two countries that have close re-lationships and effects on each other. When a country advances in technology, its (friend or) neighbor country can be influenced so that the technology is propagated among the people of that country as well. Comparing against ICA, our proposed algorithm brings a network of connected regions that help each other increase the mean power where ICA provides a set of isolated empires that are in a worldwide clash and are capable to increase their mean power only with their own colonies. Of course the empires try to occupy the colonies of the other empires to increase their power; however, such evolution is assumed to be slower than that of our net-worked infrastructure.

\section{Problem Statement}

The ICA starts by creating random empires and expanding their territories based on the imperialistic competition. Colonies are converged towards their relevant Impe-rialists according to the assimilation policy. But the colonies of an empire do not have the possibility of moving towards other powerful empires until they quit the current empire and are absorbed into the more powerful ones. Therefore, we aim to provide the possibility of moving (i.e. converging) towards other powerful empires (or Impe-rialists) for a colony without joining those empires or even before joining them. This is assumed to provide more interactions in the population component of our algo-rithm.

If we consider quorums as our Dialogue infrastructures, then our main idea is to put the countries into quorums and let them evolve in quorums. In this way, the most powerful country (i.e. Imperialist) within a quorum can be directly or indirectly ac-cessible to the colonies at the other quorums. Quorums intersect at the members (i.e. countries), which can influence the other members of quorums. Suppose an Imperial-ist is at the intersection of two quorums. Then, it can probably affect two empires at the same time. Moreover, it happens that the colonies of powerful quorums are placed at the intersection of different quorums. We assume that such a colony from a power-ful quorum (i.e. empire) can also influence weaker empires because it might have inherited power from a more powerful empire. It sounds that by organizing countries into quorums we will have more interactions between the countries in comparison to putting them into separated empires, which tries to compete each other in dominating colonies.

Overall, we assume that the policy of using Dialogue infrastructures could be used to provide a better convergence rate in comparison to the policy of ICA because of more interactions in the population component of the evolutionary algorithm. Thus, we have come to the following research question: 
Q: How could we make use of Dialogue infrastructures, such as quorums, in order to provide a better convergence rate in comparison to Imperialist Competitive Algo-rithm?

\section{Conclusions and Future work}

In conclusion, we are thinking of increasing the capability of exchanging infor-mation in the population component of the evolutionary algorithms by providing an infrastructure for Dialogues. In other words, we are thinking of dividing a population into several regions equivalent to the empires in ICA where instead of the competition among regions, the notion of Dialogue among regions is introduced, which is as-sumed to improve the convergence rate.

As future work we will complete the proposed model and apply it in a wide range of problems. We are motivated to seek the applications that the new algorithm might bring to the different areas. We therefore provide complex optimization scenarios in different majors especially in Game theory, industrial controllers and sensor networks in order to evaluate our approach and compare it against similar algorithms.

\section{References}

[1]. A. E. Eiben and J. E. Smith, Introduction to evolutionary computing vol. 2: Springer Berlin, (2010).

[2]. R. G. Reynolds, "An introduction to cultural algorithms," in Proceedings of the third annual conference on evolutionary programming, (1994), pp. 131-139.

[3]. E. Atashpaz-Gargari and C. Lucas, "Imperialist competitive algorithm: an algorithm for optimization inspired by imperialistic competition," in 2007 IEEE Congress on Evolu-tionary Computation, Singapore,(2007), pp. 4661-4667, IEEE.

[4]. E. Atashpaz-Gargari and C. Lucas, "Designing an optimal PID controller using Colo-nial Competitive Algorithm," in First Iranian Joint Congress on Intelligent and Fuzzy Sys-tems, Mashhad, Iran, (2007).

[5]. E. Atashpaz-Gargari, F. Hashemzadeh, R. Rajabioun, and C. Lucas, "Colonial com-petitive algorithm: a novel approach for PID controller design in MIMO distillation column process," International Journal of Intelligent Computing and Cybernetics, vol. 1, pp. 337- 355, (2008).

[6]. R. Rajabioun, E. Atashpaz-Gargari, and C. Lucas, "Colonial competitive algorithm as a tool for Nash equilibrium point achievement," in Computational science and its applica-tions-iccsa 2008, Perugia, Italy, ed: Springer, (2008), pp. 680-695.

[7]. M. Roshanaei, E. Atashpaz-Gargari, and C. Lucas, "Adaptive beamforming using co-lonial competitive algorithm," in 2nd International Joint Conference on Computational En-gineering, Vancouver, Canada, (2008).

[8]. A. D. Kshemkalyani and M. Singhal, Distributed computing: principles, algorithms, and systems: Cambridge University Press, (2008). 